\title{
GENETIC BACKGROUND AND EXPRESSIVITY OF CONGENITAL CATARACT IN MICE${ }^{1)}$
}

\author{
MASAYO HARATA, RYUJIRO SHOJI AND REIJI SEMBA \\ Institute for Developmental Research, Aichi Prefectural Colony \\ Kasugai, Aichi 480-03
}

Received September 8, 1977

Cataract is clinically the commonest and most important disease of the lens. Many environmental factors such as drugs, diet and X-ray irradiation which are involved in cataract formation have come to light (Kirsch 1975; van Heyningen 1976). A cataract mutant among laboratory animals will be a useful tool for elucidating the role of genetic as well as environmental factors in cataract formation. Ohtori et al. (1968) found a female mouse showing lens opacity in JCL : ICR mice and established the CTS strain with congenital cataract and small eyes. The cataract was controlled by a single autosomal dominant gene Cts, and the microphthalmia manifested only when the gene was in homozygous state (Ohtori et al. 1968). The present authors investigated the role of background genes in the morphogenesis of cataract in the Cts/+ heterozygotes. Maldevelopments of this cataractous conditions were also examined histologically through prenatal stages under a light microscope.

\section{MATERIALS AND METHODS}

Mice used were CTS (Mutant gene Cts/Cts; genet $a / a B / B c / c)$ and CTA/Idr $(C t s / C t s$; $A / A B / B c / c)$, strains bearing $C t s$ gene; C57BL/6J $(+1+; a / a B / B C / C), \mathrm{KYF} / \mathrm{MsIdr}$ $(+1+; a / a b / b C / C)$ and MT/HokIdr $(+1+; A / A B / B c / c)$, inbred strains maintained in our institute (Idr); and JCL: ICR $(+\mid+; A / A B / B c / c)$ strain from a closed colony bred by a commercial breeder (Nihon CLEA Co. Ltd., Tokyo). CTA strain was established in our institute from hybrids between CTS and ICR mice under selective inbreeding marked on the eye abnormality. Though CTA and CTS strains originated from ICR, there is a difference between them in agouti locus; wild type in CTA strain and non-agouti in CTS strain.

All animals were bred in an air-conditioned room $\left(23 \pm 2^{\circ} \mathrm{C}\right.$ in room temperature; $65 \pm 10 \%$ in humidity), and fed CMF pellets (Oriental Yeast Co. Ltd., Tokyo) and tap water ad libitum.

CTS mice were reciprocally outcrossed with KYF. Reciprocal outcrosses of CTA were made with KYF, C57BL, MT and ICR, respectively (Table 1). Some heterozygotes $(C t s /+)$ from the cross between CTA $(C t s / C t s)$ and $\mathrm{KYF}(+\mid+)$ were intercrossed with

1) Supported by a grant-in-aid for the Special Project Research (No. 111512) from the Ministry of Education, Science and Culture of Japan. 
Table 1. Fluctuation of expressivity of $C t s$ gene in heterozygous mice with different genetic backgrounds

\begin{tabular}{cccc}
\hline \hline \multirow{2}{*}{$\begin{array}{c}\text { Parents of reciprocal } \\
\text { outcross }\end{array}$} & \multicolumn{3}{c}{ No. of youngs observed } \\
\cline { 2 - 4 } & Total & "mc"* $(\%)$ & "c"* (\%) \\
\hline CTS and KYF & 129 & $118(91.5)$ & $11(8.5)$ \\
CTA and KYF & 76 & $76(100)$ & $0(0)$ \\
CTA and C57BL & 111 & $82(73.9)$ & $29(26.1)$ \\
CTA and ICR & 161 & $32(19.9)$ & $129(80.1)$ \\
CTA and MT & 85 & $6(7.1)$ & $79(92.9)$ \\
\hline
\end{tabular}

* Abbreviations explained in the text.

Table 2. Segregation of phenotype in $F_{1}$ and $F_{2}$

\begin{tabular}{|c|c|c|c|c|c|c|c|}
\hline \multirow{3}{*}{\multicolumn{3}{|c|}{ Parents of reciprocal crosses }} & \multicolumn{5}{|c|}{ No. of youngs observed } \\
\hline & & & \multicolumn{5}{|c|}{ Phenotype* } \\
\hline & & & Total & “n” & “mc" & "c" $(\%)^{* *}$ & "cmp" \\
\hline $\begin{array}{l}\text { CTA “cmp" } \\
\text { Cts/Cts }\end{array}$ & and & $\begin{array}{l}\text { KYF “n” } \\
+ \text { It }\end{array}$ & 76 & 0 & 76 & $0(0)$ & 0 \\
\hline $\begin{array}{l}\mathrm{F}_{1} \text { "mc" } \\
\text { Cts/t }\end{array}$ & and & $\begin{array}{l}\mathrm{F}_{1} \text { "mc" } \\
\text { Cts } /+\end{array}$ & 329 & 80 & 164 & $11(6.3)$ & 74 \\
\hline $\begin{array}{l}\mathrm{F}_{1} " \mathrm{mc} " \\
\text { Cts/t }\end{array}$ & and & $\begin{array}{l}\text { CTA “cmp" } \\
\text { Cts/Cts }\end{array}$ & 191 & 0 & 100 & $7(6.5)$ & 84 \\
\hline $\begin{array}{l}\mathrm{F}_{1} " \mathrm{mc} " \\
\text { Cts } /+\end{array}$ & and & $\begin{array}{l}\text { ICR "n" } \\
+ \text { It }\end{array}$ & 184 & 95 & 79 & $10(11.2)$ & 0 \\
\hline
\end{tabular}

* Phenotypic classification ("n", "mc", "c", and "cmp") given in the text.

** \% of "c" in heterozygotes ("mc" $+\mathrm{c} ")$.

themselves or backcrossed to CTA or outcrossed to ICR $(t+t)$ mice (Table 2$)$. All the offsprings derived from these crosses were inspected at least twice with the unaided eye, and once at about 40 days of age with a slit-lamp microscope after pretreatment with mydriatica Mydrin-P (Santen Pharmaceutical Co. Ltd., Osaka).

Specimens submitted to histological observations were fetuses of CTA (Cts/Cts) and JCL : ICR $(+\mid+)$ mice. They were removed from pregnant females of each strain on day $10,12,14,15$ and 16 of gestation $(v p=0)$, and immediately fixed in Bouin's solution. After fixing the preparation for about 20 days, the specimens dehydrated by $\mathrm{n}$-butyl-alcohol method were sectioned at $10 \mu \mathrm{m}$ with a routine paraffin method. Histological specimens were stained by $\mathrm{H}-\mathrm{E}$ and observed under a light microscope.

\section{RESULTS}

By inspection of eyes with a slit-lamp microscope and the unaided eye, offsprings were divided into four types according to the degree of lens opacity: (1) Normal " $n$ " 
type, in which no lens opacity was observed; (2) mild cataract "mc" type, in which a slight lens opacity was found only under slit-lamp microscopy; (3) cataract "c" type, in which lens opacity was observed not only under slit-lamp microscopy but also with the unaided eye; and (4) cataract and microphthalmia "cmp" type, in which the lens was white opaque and the eye size was only half of the normal.

The offsprings of CTS and CTA strains (Cts/Cts) were always of "cmp" type. No significant phenotypic variation was detected among them. All the offsprings of C57BL, KYF, MT and ICR strains $(+\mid+)$ were of " $n$ " type. "Mc" and "c" types were found in the cross between $C t s / C t s$ and $+1+$ strains. Thus, these two types were assumed as the phenotypic variants of $C t s /+$ heterozygotes.

The results of the cross experiments were summarized in Tables 1 and 2 . In the tables, the number of each phenotype represents the summed up number obtained in a cross and in its reciprocal cross, because there was no significant difference between them. No association of $C t s$ gene with sex, $A, B$ and $C$ loci was detected.

Frequency of "mc" and "c" types in heterozygotes fluctuated significantly, depending upon the crossed parents as shown in Table $1(\mathrm{p}<0.05)$. When KYF was reciprocally crossed with CTS and CTA, frequency of "c" type was $8.5 \%$ and $0 \%$, respectively. When CTA was crossed with KYF, C57BL, ICR, and MT, frequency of "c" type was $0 \%, 26.1 \%, 80.1 \%$, and $92.9 \%$, respectively. Though no "c" type was found in $\mathrm{F}_{1}$ from the reciprocal cross between CTA and KYF, it appeared in their $F_{2}$ (Table 2). The difference was significant ( $\mathrm{p}<0.05$ by Fisher's direct method). Frequency of "c" type in $\mathrm{F}_{2}$ heterozygotes ("mc"+ "c") was $6.3 \%, 6.5 \%$, and $11.2 \%$ when the $\mathrm{F}_{1}$ was intercrossed, backcrossed to CTA, and outcrossed to ICR, respectively.

Homozygous lenses of CTA mice at 14 prenatal days indicated normal development in histological observations. Initial changes occurred at prenatal day 15 , and lens fibers around the lenticular nucleus swelled remarkably and transformed into spindle shapes (Figs. 1 and 2). Other abnormalities observed at this stage were irregular dispositions of the lens fibrous nuclei and the unequal size of nuclei in the lens fibers. At prenatal day 16, the lens fibers usually appeared to be more swollen (Figs. 3, 4). In severely affected specimens, the degenerated lens fibers formed masses and interspaced with vacuoles, which were found mainly near the anterior pole of the lens (Fig. 5). These histological changes progressed gradually after birth. The homozygous lens, lost almost all of the normal structures, appeared a typical feature of cataract with microphthalmia at eye opening (Fig. 6). But no changes were detected in the lens epithelium and other eye organs.

\section{DISCUSSION}

Mutant mice with hereditary cataract caused by a recessive gene (Nakano et al. 1960; Tissot and Cohen 1972) or a dominant gene (Green 1966; Ohtori et al. 1968) have been reported. According to Ohtori et al. (1968), cataract of CTS mice is controlled by a single autosomal dominant gene $C t s$, which manifests lens opacity in both heterozygous and homozygous conditions. In the present experiment, all heterozygotes were 

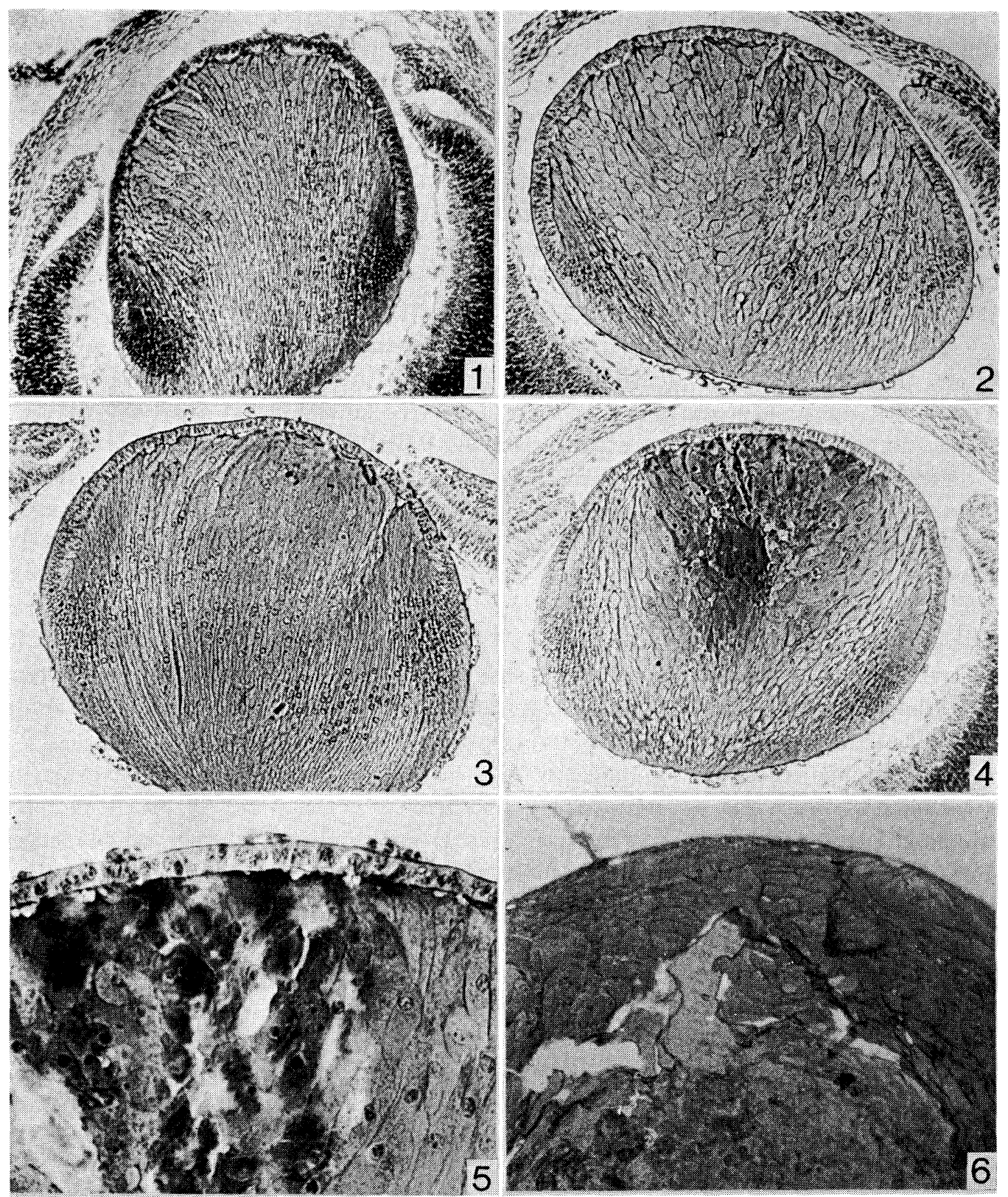

Fig. 1-6. 1. A normal lens at day 15 of fetal life; phase-contrast micrograph. 2. The swelling of lens fibers, an initial change in cataract formation in $\mathrm{Cts} / \mathrm{Cts}$ homozygotes at day 15 of fetal life; phase-contrast micrograph. 3. A normal lens at day 16 of fetal life; phase-contrast micrograph. 4. Severe swelling of fiber cells in cataractous lens at day 16 of fetal life; phase-contrast micrograph. 5. Degenerated cell masses of lens fibers and interspaced vacuoles near the anterior pole area of the Cts/Cts homozygous lens at day 16 of fetal life. 6. Cts/Cts homozygous lens lost in almost all of normal structures at eye opening of postnatal day 14 . 
determined as "mc" or "c" types (Table 1), indicating that the penetrance of the Cts gene in the Cts/+ heterozygotes was complete. However, the slip-lamp microscopy revealed that the degree of lens opacity in "mc" or "c" type was less severe than that in "cmp" type. This means that the expressivity of the Cts gene in heterozygotes is lower than that in homozygotes. Thus, it would be better to define the nature of the gene as semidominant instead of dominant. Although the numbers of "cmp" type in Table 2 are around 10\% smaller than those expected, the results in Tables 1 and 2 support the idea that the Cts gene is a single autosomal gene.

Frequency of "c" type in the Cts/+ heterozygotes fluctuated from $0 \%$ to $93 \%$, depending upon their parents (Table 1). The fluctuation in expressivity will be mostly attributable to the background genes. In the reciprocal crosses between CTA and KYF, frequency of "c" type in the $C t s /+$ heterozygotes was lower in $F_{1}$ than in $F_{2}$ from the $\mathrm{F}_{1}$ intercross. According to the multifactorial/threshold model, a change in frequency of the malformation can result from either a shift of the distribution or a change in variance (Fraser 1976). In the present experiment, no shift of the genetic distribution would occur between $F_{1}$ and $F_{2}$. Thus, it is suggested that the variance of the genetic constituents in $F_{2}$ may be greater than that in $F_{1}$. This presumption coincides with the fact that the genetic constituents are heterogeneous in $F_{2}$ while they are homogeneous in $F_{1}$. The findings also suggest the effect of background genes on the expressivity of the $C t s$ gene.

On the other hand, all the strains except for ICR are inbred strains. Therefore, the genetic constituents of their $F_{1}$ are uniform in any cross. In spite of the homogeneous genetic constituents, phenotype of $F_{1}$ was not uniform in any cross. Appearance of phenotypic variants, "mc" and "c" types, in $\mathrm{F}_{1}$ suggests that some environmental factors may also affect the expressivity of the Cts gene.

The present study revealed that the $C t$ s gene possessed a complete penetrance, as would be expected of an autosomal semidominant gene, and the expressivity in the heterozygous condition varied, depending partly upon environmental factors and partly upon different backgrounds of gene constitutions. These findings may be helpful in understanding the phenotypic variation of the mutant character.

Onset of cataract formation in mice has been described differently with each mutant strain; some put it at the embryonic stage in Cat-dominant mice (Paget and Baumgartner-Gamauf 1961), others at postnatal day 14 (Brown et al. 1970) or 20-25 (Hamai et al. 1973) in cac/cac mice. The lens opacity in Cts/Cts homozygotes was recognized at about 13 to 14 days after birth (Ohtori et al. 1968), but the initial change relevant to eye organs and stages was not mentioned before.

In our histological study, the initial change of eyes was recognized at prenatal day 15 as the swelling of lens fibers in $C t s / C t s$ homozygotes. At prenatal day 16, the degeneration of these fiber cells progressed and the lens tissues were initially destroyed at the anterior pole area. The similar damage in the lens fiber cells was also demonstrated in the cataractous lens from $c a c / c a c$ homozygotes. However, the initial area of degeneration in the $c a c / c a c$ homozygotes was found in the deep posterior suture area of the perinuclear zone (Hamai et al. 1974) and in the distal region of the cortex (Brown et al. 1970). 


\section{SUMMARY}

Cataractous mice (Cts/Cts homozygotes) were reciprocally crossed with 4 inbred strains of mice. In $F_{1}$, expressivity of the gene fluctuated depending upon the background genes. Expressivity of the gene in $F_{1}$ was different from that in their $F_{2}$, reflecting the heterogeneous genetic constitution of the latter. The Cts gene possessed complete penetrance, as might be expected of an autosomal semidominant gene. The initial sign of cataract formation was found at prenatal day 15 in the lens fibers near the anterior pole area of $\mathrm{Cts} / \mathrm{Cts}$ homozygotes.

\section{LITERATURE CITED}

Brown, E. R., T. Nakano, and G. L. Vankin, 1970 Early development of inherited cataract in mice. Exp. Animals 19: 95-100.

Fraser, F. C., 1976 The multifactorial/threshold concept-Uses and misuses. Teratology 14: 267280.

Green, M. C., 1966 Mutant genes and linkages. In "Biology of the Laboratory Mouse" (E. L. Green, 2nd ed.) pp. 87-150, McGraw-Hill, New York.

Hamai, T., H. N. Fukui, and T. Kuwabara, 1974 Morphology of hereditary mouse cataract. Exp. Eye Res. 18: 537-546.

Kirsch, R. E., 1975 The lens. Arch. Ophthalmol. 93: 284-314.

Nakano, K., S. Yamamoto, G. Kutsukake, H. Ogawa, A. Nakajima, and E. Takano, 1960 Here ditary cataract in mice. Japan. J. Clin. Ophthalmol. 14: 1772-1776.

Ohtori, H., T. Yoshida, and T. Inuta, 1968 "Small eye and cataract", a new dominant mutation in the mouse. Exp. Animals 17: 91-96.

Paget, O. E., and M. Baumgartner-Gamauf, 1961 Histologische Untersuchungen an einer dominant erblichen Form einer Cataract bei der Hausmaus. Zool. Anz. 166: 55-69.

Tissot, R. G., and C. Cohen, 1972 A new congenital cataract in the mouse. J. Hered. 63: 197201.

van Heyningen, R., 1976 Experimental studies on cataract. Invest. Ophthalmol. 15: 685-697. 